\title{
Cross-coupling ketones
}

J. Am. Chem. Soc. 140, 5347-5341 (2018)
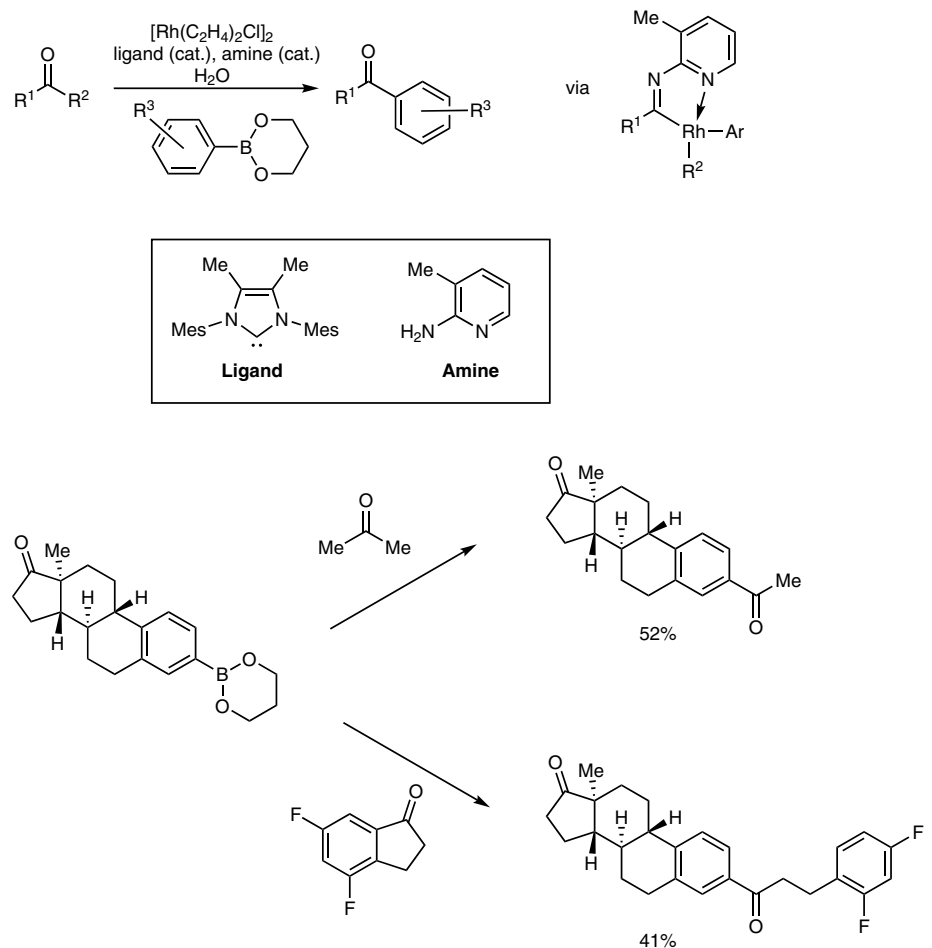

The Suzuki-Miyaura coupling has long been a go-to reaction for the formation of carbon-carbon bonds. From its early history as a method to form Cs $p^{2}-\mathrm{Cs} p^{2}$ bonds, continual developments have allowed it to remain at the forefront of synthetic chemistry, including the ability to work with $s p^{3}$-hybridized carbons and - largely thanks to nickel catalysis - the ability to employ a broad range of electrophiles beyond aryl and alkyl halides. Now Guangbin Dong and co-workers at the University of Chicago have expanded the electrophile scope even further by allowing cross-coupling on a wide range of simple, unstrained ketones via a

\section{$\mathrm{C}-\mathrm{C}$ bond cleavage.}

Prior work had shown that this was possible, though generally very specific starting ketones were needed: those containing suitable activating/directing groups or those with significant ring strain. In the current work a wide range of ketones both cyclic and acyclic - proved capable of cross-coupling with arylboronates (pictured, top). The proposed mechanism involves the in situ formation of an intermediate imine with the amine additive, leading to coordination and subsequent insertion of the $\mathrm{Rh}(\mathrm{I})$ catalyst into the $\mathrm{C}-\mathrm{C}$ bond.
Substituted cyclopentanones were ring-opened to give alkyl aryl ketones in reasonable yields, and with unsymmetrical substrates there was a slight preference for cleavage of the more sterically hindered C-C bond. In cases where aryl alkyl ketones were used as starting materials there was exclusive cleavage of the $\mathrm{C}(\operatorname{aryl})-\mathrm{C}$ (carbonyl) bond. Dialkyl ketones such as acetone also worked with moderate yields. With respect to the boronate component ortho-substituted arylbornates were not efficient nucleophiles - presumably due to steric hindrance. However the system was tolerant of meta- and para-substituents, including di- and tri-substituted variants. Late-stage functionalization of more complex molecules was also demonstrated. Using boronate-functionalized steroid derivatives the group showed that ketones could be used as effective acylating reagents (pictured, bottom).

\section{Enda Bergin}

Published online: 11 May 2018

https://doi.org/10.1038/s41929-018-0087-4 